\title{
SIRT1 activation ameliorates hyperglycaemia by inducing a torpor-like state in an obese mouse model of type 2 diabetes
}

\author{
Richard E. Gilbert • Kerri Thai • Suzanne L. Advani • \\ Carolyn L. Cummins • David M. Kepecs • Stephanie A. Schroer • \\ Minna Woo $\cdot$ Yanling Zhang
}

Received: 29 July 2014 / Accepted: 8 December 2014 / Published online: 7 January 2015

(C) Springer-Verlag Berlin Heidelberg 2014

\begin{abstract}
Aims/hypothesis Nutrient overabundance and diminished physical activity underlie the epidemic of obesity and its consequences of insulin resistance and type 2 diabetes. These same phenomena, obesity and insulin resistance, are also observed in mammals as they ready themselves for the nutrient deprivation of winter, yet their plasma glucose does not rise. Given the role of silent information regulator 2 (Sir2) and its mammalian orthologue, Sirt1, in survival and life extension during energy deprivation, we hypothesised that enhancing its activity may reduce the insensible energy loss engendered by hyperglycaemia and glycosuria.

Methods At 8 weeks of age, $d b / d b$ and $d b / m$ mice were randomised to receive the SIRT1 activator SRT3025 milled in chow $(3.18 \mathrm{~g} / \mathrm{kg})$ or regular chow and followed for a further 12 weeks.

Results When compared with vehicle, SIRT1 activation greatly improved glycaemic control, augmented plasma insulin concentrations, increased pancreatic islet beta cell mass and elevated hepatic expression of the beta cell growth factor, betatrophin in $d b / d b$ mice. Despite the dramatic reduction in
\end{abstract}

Electronic supplementary material The online version of this article (doi:10.1007/s00125-014-3485-4) contains peer-reviewed but unedited supplementary material, which is available to authorised users.

R. E. Gilbert $(\bowtie) \cdot$ K. Thai $\cdot$ S. L. Advani $\cdot$ D. M. Kepecs $\cdot$ Y. Zhang

Keenan Research Centre for Biomedical Science and Li Ka Shing

Knowledge Institute of St Michael's Hospital, 61 Queen Street East,

Toronto, ON, Canada M5C 2T2

e-mail: richard.gilbert@utoronto.ca

C. L. Cummins

Faculty of Pharmacy, University of Toronto, Toronto, ON, Canada

S. A. Schroer $\cdot$ M. Woo

Toronto General Research Institute and Department of Medicine,

University Health Network, Toronto, ON, Canada hyperglycaemia, $d b / d b$ mice displayed worsening insulin resistance, diminished physical activity and further weight gain. These findings along with reduced food intake and reduction in body temperature resembled torpor and hibernation. By contrast, SIRT1 activation conferred only minimal changes in non-diabetic $d b / m$ mice.

Conclusions/interpretation While reducing hyperglycaemia and promoting beta cell expansion, enhancing the activity of SIRT1 facilitates a phenotypic change in a $d b / d b$ mouse model of diabetes to one that more closely resembles the physiological state of torpor or hibernation.

Keywords Betatrophin · Diabetes $\cdot$ Hepatic steatosis · Hibernation · Insulin resistance · Insulin secretion · Sirtuin . Torpor

$\begin{array}{ll}\text { Abbreviations } \\ \text { CD36 } & \text { Cluster differentiation } 36 \\ \text { FAS } & \text { Fatty acid synthase } \\ \text { FOXO1 } & \text { Forkhead box O1 } \\ \text { FOXO6 } & \text { Forkhead box O6 } \\ \text { NPY } & \text { Neuropeptide Y } \\ \text { RER } & \text { Respiratory exchange ratio } \\ \text { Sir2 } & \text { Silent information regulator 2 } \\ \text { Sirt1 } & \text { Sirtuin 1 } \\ \text { SREBP-1c } & \text { Sterol regulatory element-binding protein-1c } \\ \text { TRH } & \text { Thyrotophin releasing hormone }\end{array}$

\section{Introduction}

The epidemic of obesity is frequently attributed to the dual adverse effects of nutrient overabundance and 
diminished physical activity. While the vast majority of younger and middle-aged individuals are able to maintain normoglycaemia in the face of obesity-induced insulin resistance by a compensatory increase in glucosestimulated insulin secretion, this ability diminishes with age so that dysglycaemia and diabetes ensue $[1,2]$. Accordingly, obesity and the insulin resistance that it engenders are viewed as inexorably linked pathophysiological states of the modern human era that underlie the rapid increase in the incidence of type 2 diabetes. These same phenomena are paradoxically also observed in hibernating mammals as they ready themselves for the nutrient deprivation of winter. Indeed, during this preparatory phase, animals typically double their body weight [3] and increase their circulating insulin concentration more than fourfold [4]. However, despite these dramatic changes, plasma glucose levels in these animals do not rise [5].

Given the role of silent information regulator $2(\mathrm{Sir} 2)$ and its mammalian orthologue, Sirt1, in allowing for survival and life extension in the setting of energy deprivation [6-9], we hypothesised that enhancing the activity of Sir2 would enable a transition from the obese, insulin-resistant diabetic phenotype to one that more closely resembles mammalian torpor or hibernation. With this in mind, we studied the $d b / d b$ mouse, a rodent model that develops obesity, insulin resistance and ultimately beta cell failure to recapitulate many of the features of type 2 diabetes in humans. In addition, we took advantage of a new generation of SIRT1activating compounds currently in clinical development that act by accelerating the SIRT1 catalysed deacetylation $[10,11]$.

\section{Methods}

Animal experiments Thirty-six 6-week-old male $d b / d b$ (BKS.Cg-Dock7m +/+ Leprdb/J) mouse models of diabetes and 12 age-matched $\mathrm{db} / \mathrm{m}$ (Dock $7 \mathrm{~m}+/+\mathrm{Leprdb}$, heterozygote from the same colony) mice were purchased from Harlan Laboratories (Indianapolis, IN, USA). At 8 weeks of age, the $d b / d b$ and $d b / m$ mice were randomised to receive the SIRT1 activator, SRT3025 [12, 13] (gift of Sirtris/Glaxo Smith-Kline, Collegeville, Pennsylvania, USA) milled in chow $(3.18 \mathrm{~g} / \mathrm{kg})$ or regular chow.

Mice were housed in a temperature-controlled room $\left(22^{\circ} \mathrm{C}\right)$ with a $12 \mathrm{~h}: 12 \mathrm{~h}$ light-dark cycle and free access to food and water at the St Michael's Hospital Animal Research Vivarium (Toronto, ON, Canada). After 12 weeks of treatment, animals were killed at the end of a $12 \mathrm{~h}$ dark (feeding) period when urine and blood samples were collected and multiple organs, including liver, pancreas and brain were harvested and either fixed in formalin or snap frozen and stored at $-80^{\circ} \mathrm{C}$. The mediobasal hypothalamus was subsequently isolated as previously described [14].

All animal studies were approved by the St Michael's Hospital Animal Ethics Committee in accordance with the Guide for the Care and Use of Laboratory Animals (National Institutes of Health Publication No. 85-23, revised 1996).

Metabolic studies and hormone measurements Metabolic studies and hormone measurements were undertaken as previously reported [15] with measurements of blood glucose, food intake, $\mathrm{HbA}_{1 \mathrm{c}}$, insulin, C-peptide and free $\mathrm{T} 3$. The HOMA-IR was used to determine insulin resistance. Insulin tolerance tests were performed at the beginning of the 12th week on fasted animals [16]. See electronic supplementary material (ESM) Methods.

Indirect calorimetry, activity and temperature The metabolic rate, motion and respiratory exchange ratio (RER) of mice were measured by indirect calorimetry as previously reported [17], as was body temperature [18]. See ESM Methods.

Histochemistry and immunohistochemistry Pancreases and livers were fixed, processed, stained and quantified as previously reported [19]. See ESM Methods.

\section{Real-time quantitative RT-PCR}

Real-time quantitative RT-PCR was used to determine the relative expression levels of mRNAs. See ESM Methods.

Western blot The in vivo lysine deacetylase activity of SRT3025 was assessed by examining kidney tissue where protein acetylation is constitutively present in diabetic animals [20]. See ESM Methods.

Insulin sensitivity was assessed in liver tissue by immunoblotting for phosphorylated and total insulin receptor substrate with antibodies directed against phosphorylated IRS-1/2 and total IRS-1 (sc-17195 and sc8038, respectively; Santa Cruz Biotechnology, Dallas, TX, USA). SIRT1 abundance was similarly assessed in liver by immunoblot (antibody no. 2028, Cell Signaling, Boston, MA, USA) with equivalent protein loading determined by beta actin. See ESM Methods.

Statistics Data are expressed as means \pm SEM. Betweengroup differences were analysed by one way ANOVA with Fisher's Protected Least Significant Difference test post hoc. All statistics were performed using GraphPad Prism 5 for Mac OS X (GraphPad Software Inc, San Diego, CA, 
USA). A $p$ value of $<0.05$ was considered statistically significant.

\section{Results}

Animal characteristics Diabetic $d b / d b$ mice gained substantially more weight than $d b / m$ mice during the study period with $d b / d b$ mice that had received the SIRT1 activator, SRT3025, gaining more weight than mice not receiving the compound (Table 1). Despite their greater weight gain, food intake among SRT3025-treated $\mathrm{db} / \mathrm{db}$ mice was lower than that of their untreated counterparts.

Immunoblot Consistent with its mechanism of action as a lysine deacetylase, SRT3025-treated animals displayed substantially reduced lysine acetylation in the in vivo setting compared with their untreated counterparts (ESM Fig. 1).

SIRT1 protein was detected in the livers of all four animal groups but in greater abundance in those $d b / d b$ mice that had received SRT3025 (ESM Fig. 2).

Energy expenditure, body temperature and activity SIRT1 activation lowered energy expenditure in both $\mathrm{db} / \mathrm{m}$ and $\mathrm{db} /$ $d b$ mice, and this was most evident during the dark phase (Fig. 1). Reduction in body temperature was also evident but was lower in $d b / d b$ mice receiving SRT3025 where a prominent circadian pattern was also noted. Physical activity was reduced in $d b / d b$ mice but lowered further in animals that received SRT3025 compared with their non-treated counterparts. While this may have been in part due to the greater body mass of mice receiving the compound, SIRT1 activation similarly lowered activity in $\mathrm{db} / \mathrm{m}$ mice whose weight was unchanged.

When compared with untreated $d b / d b$ mice, those that had received SRT3025 showed a higher RER throughout a $24 \mathrm{~h}$ period (Fig. 1).
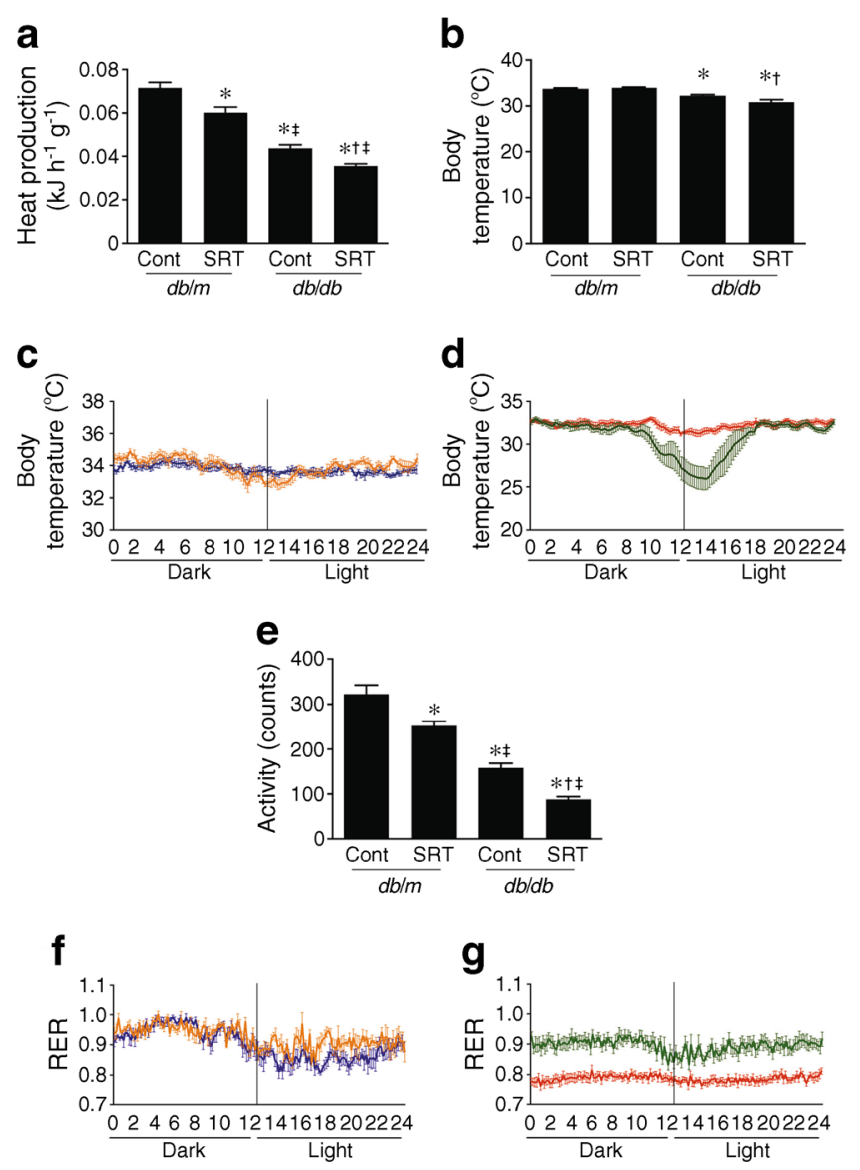

Fig. 1 (a) Heat production was reduced in $d b / d b$ compared with nondiabetic $d b / m$ mice and reduced in both animal groups by the administration of SRT3025 (SRT). (b) Body temperature was lower in $d b / d b$ than in $d b / m$ mice and fell further in $d b / d b$ mice that had received SRT3025. (c), d) A circadian pattern in body temperature was noted in $\mathrm{db} / \mathrm{m}$ and to a greater extent in $d b / d b$ mice that had received SRT3025, whereby body temperature began to dip during the latter part of the dark phase, returning to its baseline in the early hours of the light phase. (e) Activity was reduced in $d b / d b$ compared with non-diabetic $d b / m$ mice, and reduced in both animal groups by the administration of SRT3025. (f, g) RER was increased in $d b / d b$ mice that had received SRT3025 compared with untreated diabetic mice. $d b / m$ control, blue; $d b / m+$ SRT3025, orange; $d b / d b$ control, red; $d b / d b+$ SRT3025, green. ${ }^{*} p<0.05$ vs $d b / m$ control, ${ }^{\dagger} p<0.05$ vs $d b / d b$ control, ${ }^{\star} p<0.05$ vs $d b / m+$ SRT3025

Table 1 Animal characteristics

\begin{tabular}{lllll}
\hline & $d b / m$ & $d b / m+$ SRT3025 & $d b / d b$ & $d b / d b+$ SRT3025 \\
\hline BW (g) & $32.7 \pm 0.2$ & $28.9 \pm 0.2$ & $42.6 \pm 0.4^{*}$ & $50.1 \pm 0.3^{* \dagger}$ \\
Food intake (mg gBW ${ }^{-1}$ day $\left.^{-1}\right)$ & $113.4 \pm 1.1$ & $130.8 \pm 2.3$ & $191.0 \pm 1.8^{*}$ & $80.2 \pm 1.2^{* \dagger}$ \\
Urine output (ml/day) & $3.0 \pm 0.1$ & $2.9 \pm 0.1$ & $18.5 \pm 0.6^{*}$ & $2.5 \pm 0.7^{* \dagger}$ \\
Fasting glucose (mmol/l) & $5.4 \pm 0.2$ & $6.8 \pm 0.2$ & $29.0 \pm 2.9^{*}$ & $15.4 \pm 0.6^{* \dagger}$ \\
Non-fasting glucose (mmol/l) & $7.2 \pm 0.1$ & $6.2 \pm 0.1$ & $33.3 .3 \pm 0^{*}$ & $14.3 \pm 0.3 * \dagger$ \\
HbA $_{1 \mathrm{c}}(\%)(\mathrm{mmol} / \mathrm{mol})$ & $4.1 \pm 0.1(21 \pm 1.1)$ & $4.0 \pm 0.1(20 \pm 1.1)$ & $11.7 \pm 0.1^{*}(104 \pm 1.1)$ & $5.3 \pm 0.1^{* \dagger}(34 \pm 1.1)$ \\
\hline
\end{tabular}

$\mathrm{BW}$, body weight

${ }^{*} p<0.05$ vs $d b / m$ mice; ${ }^{\dagger} p<0.05$ vs $d b / d b$ mice 

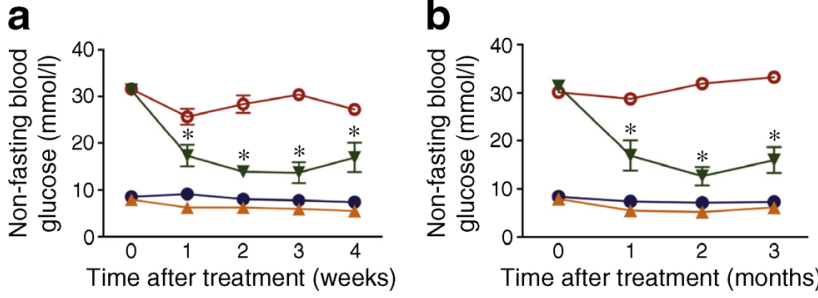

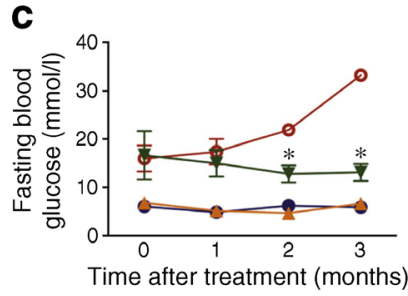

Fig. 2 (a, b) Non-fasting and (c) fasting blood glucose concentrations. Non-fasting blood glucose was lower in SRT3025-treated $d b / d b$ mice after 1 week, while fasting glucose became lower by the second month of the study. Non-diabetic $d b / m$ mice were unaffected by drug treatment. $d b /$ $m$ control, blue; $d b / m+$ SRT3025 orange; $d b / d b$ control, red; $d b / d b+$ SRT3025, green. ${ }^{*} p<0.01$ vs $d b / d b$ control mice
Plasma free T3 concentrations differed between $d b / m$ and $d b / d b$ mice but were unaffected by SRT3025 treatment (ESM Fig. 3).

Sirt1 activation ameliorates hyperglycaemia in $d b / d b$ mice Within 1 week of receiving SRT3025, non-fasting blood glucose levels were lower in treated $d b / d b$ mice in comparison with $d b / d b$ mice that had received regular chow (Fig. 2). Fasting glucose levels were also lower in SRT3025-treated $d b / d b$ mice, although this only became manifest after 4 weeks. Both SRT3025 and regular chow-treated $\mathrm{db} / \mathrm{m}$ mice remained normoglycaemic throughout the study, with no difference in blood glucose between the two groups in either the fasting or non-fasting states (Fig. 2).

In alignment with the blood glucose findings, $\mathrm{HbA}_{1 \mathrm{c}}$ measured after 4 weeks and at the end of the study was approximately twofold higher in untreated $d b / d b$ mice but approached levels of normoglycaemic $\mathrm{db} / \mathrm{m}$ animals in $d b / d b$ mice that had received SRT3025. HbA 1 levels were unaffected by SRT3025 in non-diabetic $\mathrm{db} / \mathrm{m}$ mice (Table 1).

Plasma insulin and insulin resistance are increased with Sirt1 activation in $d b / d b$ mice At 6 weeks of age, $d b / d b$ mice are characteristically obese and insulin resistant, maintaining normoglycaemia by compensatory hyperinsulinaemia. By 10-14 weeks, however, the circulating insulin concentration falls and blood glucose rises. As expected, we observed these changes in untreated $d b / d b$ mice. By contrast, animals that had received SRT3025 had markedly augmented plasma insulin concentrations in relation to their untreated counterparts (Fig. 3). However, the C-peptide:insulin ratio was reduced in these animals suggesting that diminished hepatic clearance of insulin may also contribute to the hyperinsulinaemia seen in these animals (ESM Fig. 4).

When compared with untreated $d b / d b$ mice those that had received SRT3025 displayed increased insulin resistance as indicated by the results of the insulin tolerance test, HOMAIR and measurement of hepatic phosphorylated IRS-1 (Fig. 3, ESM Fig. 5).

Gene expression In the setting of insulin resistance, impaired insulin signalling fails to repress hepatic gluconeogenesis, thereby contributing to fasting hyperglycaemia in type 2 diabetes. Consistent with the insulin resistance of the $d b / d b$ mouse, expression of the key gluconeogenic enzymes Pepck and G6pc (also known as G6Pase) mRNA was increased approximately fivefold in $d b / d b$ mice when compared with $\mathrm{db} / \mathrm{m}$ controls. By contrast, in mice that had received SRT3025 the overexpression of gluconeogenic enzymes was substantially reduced, consistent with the ability of high insulin concentrations to overcome insulin resistance (Fig. 4).

Expression of the lipogenic enzyme fatty acid synthase (FAS), but not the fatty acid transporter cluster differentiation 36 (CD36) was increased in SRT3025-treated $d b / d b$ mice (Fig. 4).

Consistent with a shift from gluconeogenesis to lipogenesis, changes in mRNA levels of related transcription factors
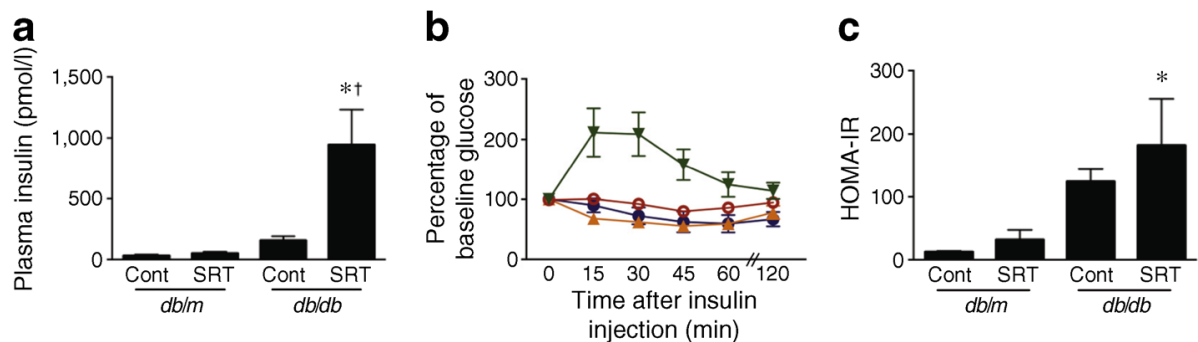

Fig. 3 (a) Fasting plasma insulin, (b) insulin tolerance test and (c) HOMA-IR. When compared with untreated $d b / d b$ mice, those that had received SRT3025 (SRT) had higher plasma insulin, an increase in plasma glucose in response to insulin administration and higher
HOMA-IR. Non-diabetic $d b / m$ mice were unaffected by drug treatment. ${ }^{*} p<0.01$ vs control $d b / m$ mice; ${ }^{\dagger} p<0.01$ vs control $d b / d b$ mice $d b / m$ control, blue; $d b / m+$ SRT3025 orange; $d b / d b$ control, green; $d b / d b+$ SRT3025, red 

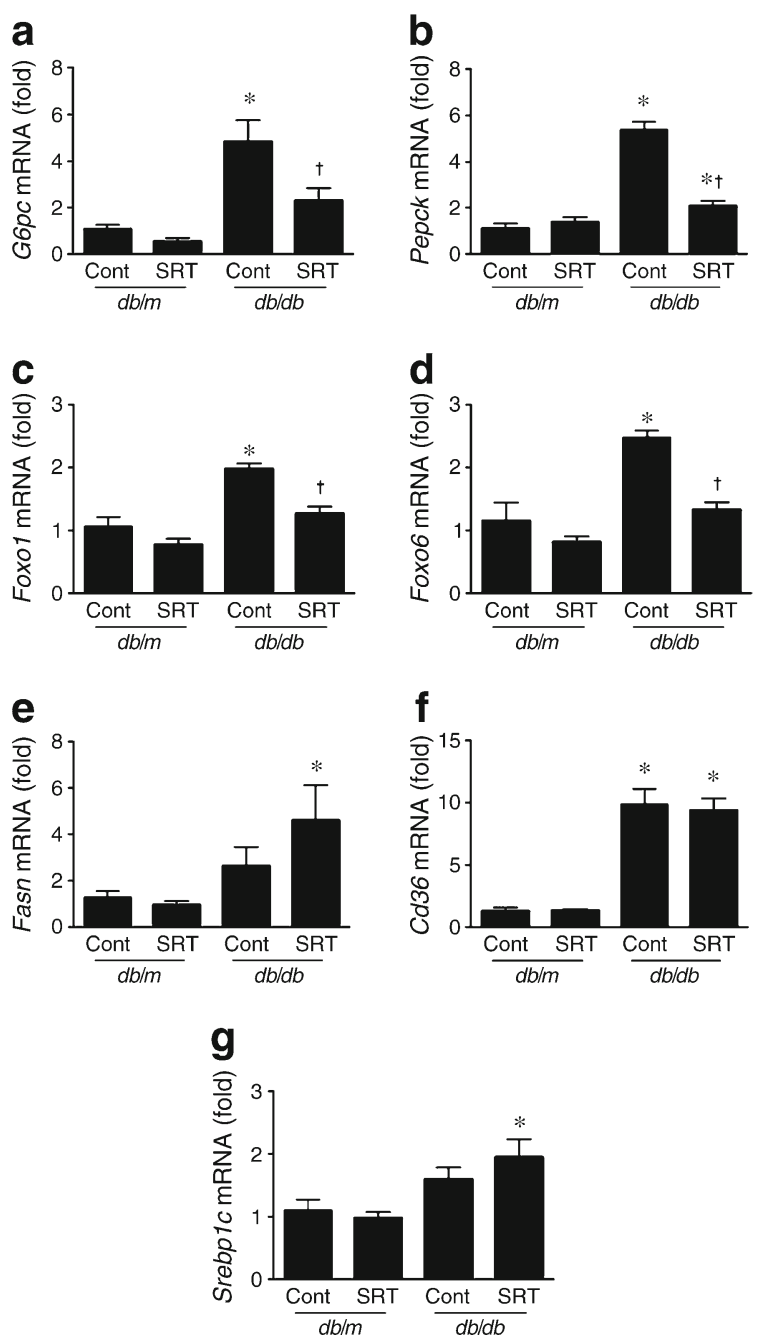

Fig. 4 Liver gene expression of (a-d) key gluconeogenic enzymes and insulin-suppressible transcription factors that initiate gluconeogenic gene transcription, G6pc, Pepck, Foxol and Foxo6, and (e-g) factors associated with fat deposition, Fasn, the fatty acid transporter $C d 36$ and Srebp1c. mRNA abundance was expressed relative to that of RPLI3a. The ratio, so-derived, was then expressed as fold change relative to that in $\mathrm{db} / \mathrm{m}$ control mice that was arbitrarily set at $1 . G 6 p c$ and Pepck mRNA were increased approximately fivefold and Foxol and Foxo6 mRNA were increased approximately twofold in $d b / d b$ mice compared with $d b /$ $m$ controls. SRT3025 (SRT) administration led to a reduction in this overexpression in $d b / d b$ mice to levels similar to those seen in nondiabetic $\mathrm{db} / \mathrm{m}$ mice and that were unaffected by drug administration. ${ }^{*} p<0.01$ vs control $d b / m$ mice; ${ }^{\dagger} p<0.01$ vs control $d b / d b$ mice

forkhead box O1 (Foxo1), forkhead box O6 (Foxo6) and sterol regulatory element-binding protein-1c $(S r e b p-1 c)$ were also found. Here, the diabetes-associated overexpression of the gluconeogenic Foxo transcription factors was reduced with SRT3025 (Fig. 4).

SIRT1 activation had no effect on gene expression of any of the gluconeogenic or lipogenic enzymes, or on transcription factors in $d b / m$ mice (Fig. 4).

In the hypothalamus, gene expression of Npy and Trh was reduced in both untreated and treated diabetic mice when compared with their non-diabetic $d b / m$ counterparts. Pomc and Agrp mRNA, on the other hand, were similar in all four groups (ESM Fig. 6).

Sirt1 activation exacerbates hepatic steatosis Non-alcoholic liver disease is closely associated with obesity, insulin resistance and type 2 diabetes in population studies and while excessive fat deposition (steatosis) is found in the livers of $d b / d b$ mice it does not progress to steatohepatitis or cirrhosis [21]. When compared with lean $d b / m$ mice, the livers of $d b / d b$ mice showed marked lipid deposition when stained with Oil Red O (Fig. 5). A further, substantial increase in the extent of hepatic steatosis was seen in animals that had received the SIRT1 activator, SRT3025. In line with these findings, $d b / d b$ mice that had received the compound showed increased hepatic expression of the transcription factor SREBP-1c and FAS but not the fatty acid transporter CD36 compared with their non-treated counterparts (Fig. 4).

Beta cell mass is increased with Sirt1 activation in $d b / d b$ mice Early in their development, $d b / d b$ mice display islet
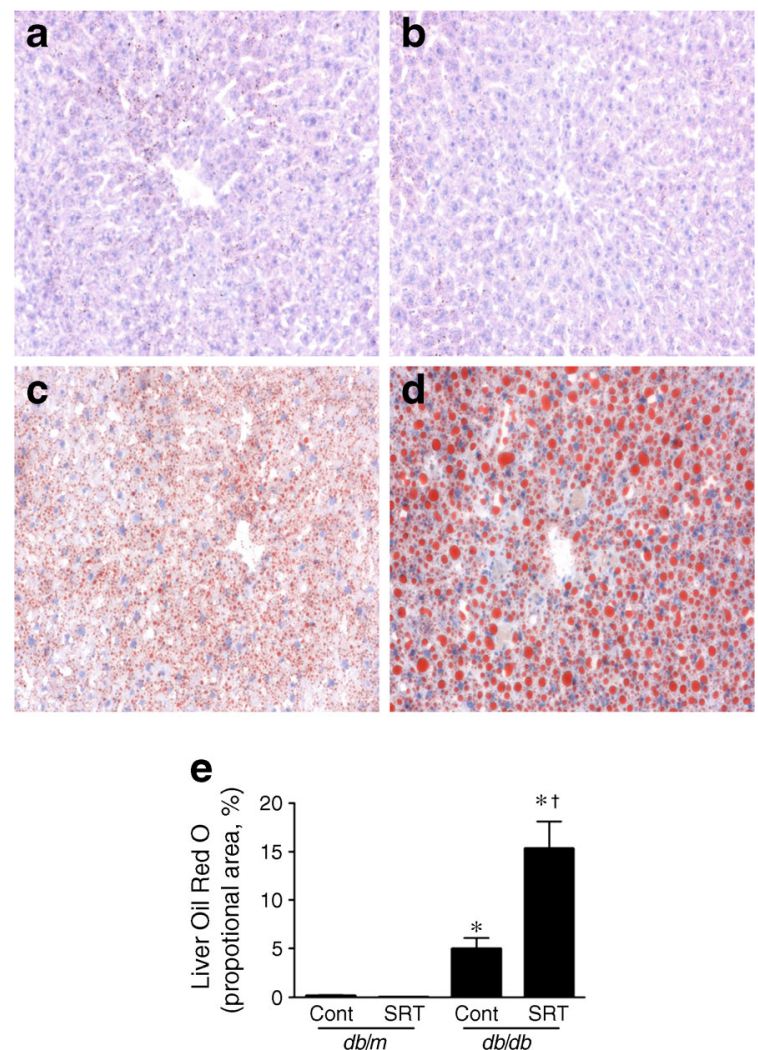

Fig. 5 Liver from non-diabetic $d b / m$ mice treated with (a) vehicle or (b) SRT3025 (SRT) and diabetic $d b / d b$ mice treated with (c) vehicle or (d) SRT3025 stained for neutral lipid with Oil Red O, and (e) their quantification. $d b / d b$ mice show abundant lipid deposition that is further increased in those that had received SRT3025. Non-diabetic $\mathrm{db} / \mathrm{m}$ mice had minimal lipid that was unaffected by drug. Magnification $\times 160$. ${ }^{*} p<0.01$ vs control $d b / m$ mice; ${ }^{\dagger} p<0.01$ vs control $d b / d b$ mice 
enlargement allowing them to maintain normoglycaemia in the face of insulin resistance. Subsequently, however, the expanded islets involute leading to relative insulin deficiency and hyperglycaemia. When compared with untreated, agematched $d b / d b$ mice, SRT3025-treated $d b / d b$ mice displayed increased beta cell mass, predominantly as a consequence of islet enlargement (Fig. 6). No effect of SRT3025 on beta cell mass was noted in non-diabetic $d b / m$ mice.

Betatrophin Betatrophin is a recently identified peptide hormone, primarily expressed in liver and fat, which promotes pancreatic beta cell proliferation and expansion of beta cell mass to improve glucose tolerance [22]. While no difference in hepatic betatrophin expression was evident between untreated $d b / d b$ and $d b / m$ mice, expression was elevated in $d b / d b$ mice that had received SRT3025 (Fig. 7).

\section{Discussion}

Obesity accompanied by insulin resistance and diabetes is commonly viewed as a consequence of excessive food consumption and reduced physical activity, often erroneously
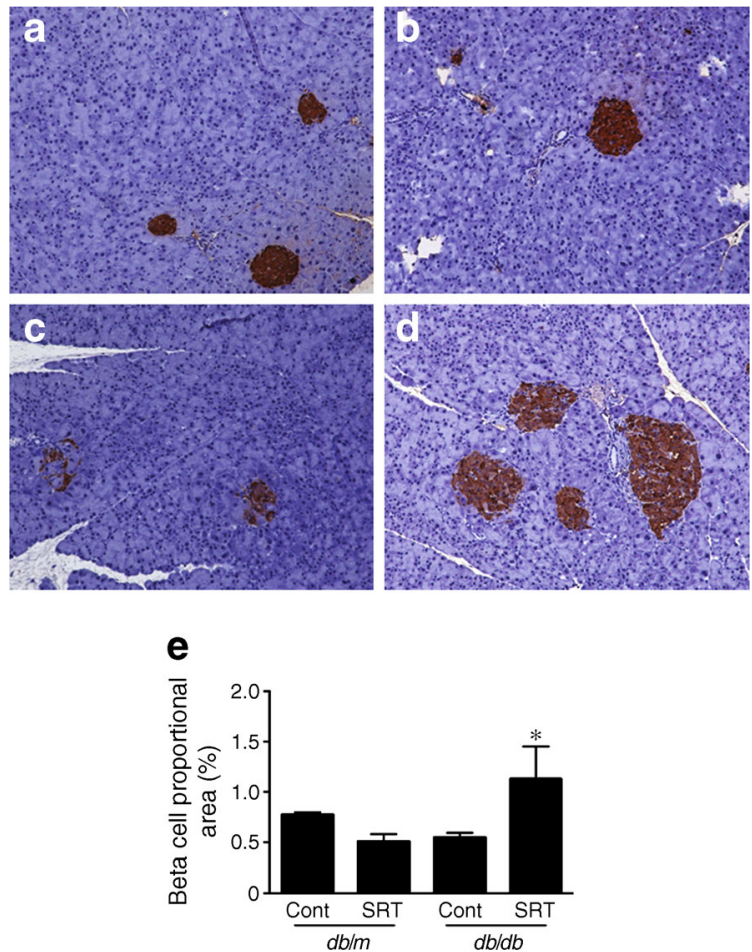

Fig. 6 Pancreases showing immunolabelled insulin in non-diabetic $\mathrm{db} / \mathrm{m}$ mice treated with (a) vehicle or (b) SRT3025 (SRT) and diabetic $d b / d b$ mice treated with (c) vehicle or (d) SRT3025, and (e) their quantification. While the islets of $d b / d b$ mice show reduction in immunostainable insulin (brown) the reverse is seen in those that had received SRT3025. Nondiabetic $\mathrm{db} / \mathrm{m}$ mice were unaffected by drug treatment. Magnification $\times 160 .{ }^{*} p<0.01$ vs control $d b / d b$ mice

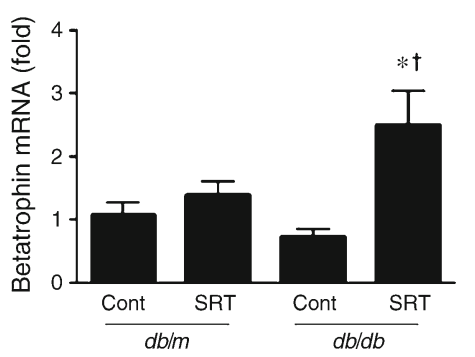

Fig. 7 Betatrophin expression in the livers of experimental animals. mRNA abundance was expressed relative to that of RplI3a. The ratio, so-derived, was then expressed as fold change relative to that in $\mathrm{db} / \mathrm{m}$ control mice that was arbitrarily set at 1 . Betatrophin expression was increased in the livers of $d b / d b$ mice that had received SRT3025 (SRT) compared with untreated $d b / d b$ and $d b / m$ mice. ${ }^{*} p<0.01$ vs control $d b / m$ mice; ${ }^{\dagger} p<0.01$ vs control $d b / d b$ mice

attributed to a lack of will power and discipline. Notably, individuals with type 2 diabetes who lose relatively modest amounts of weight experience substantially improved insulin sensitivity and reductions in blood glucose concentrations. Here we show, however, that obesity, insulin resistance and hyperglycaemia are not inextricably intertwined. While pharmacological activation of SIRT1 lowered blood glucose to near-normal levels, these changes were not accompanied by weight loss and improved insulin sensitivity in $d b / d b$ mice. Indeed, the reverse occurred with augmented insulin resistance and further weight gain during the study. Rather, blood glucose lowering appeared to be a consequence of substantially increased circulating insulin concentrations and augmented pancreatic islet beta cell mass in association with elevated hepatic expression of the beta cell growth factor, betatrophin [22].

Although imperfect, the $d b / d b$ mouse recapitulates many of the features of type 2 diabetes in humans. As a consequence of a point mutation in the receptor for the anorexogenic adipokine, leptin, homozygous $d b / d b$ mice are hyperphagic, obese and insulin resistant, developing hyperinsulinemia in an attempt to maintain glucose homeostasis. As it ages, however, islet beta cell mass becomes reduced so that relative insulin insufficiency and hyperglycaemia ensue. As such, this mouse model resembles obesity with type 2 diabetes in humans where insulin and leptin resistance, rather than their deficiencies, are commonly found [23]. In the present study, the otherwise natural history of beta cell failure in the $d b / d b$ mouse was averted by SIRT1 activation. Instead of the agedependent diminution in insulin secretion, mice treated with SIRT1 activator maintained high levels of insulin that were near-commensurate with their requirements, reducing hepatic gluconeogenesis, lowering plasma glucose and achieving an $\mathrm{HbA}_{1 \mathrm{c}}$ that was approximately half that of untreated $d b / d b$ mice and only slightly higher than non-diabetic $d b / m$ mice.

Pancreatic islet beta cells constitutively express SIRT1 that, when upregulated, increase glucose-stimulated insulin secretion in both the cell culture and in vivo settings [24, 25]. The present study suggests that another SIRT1-dependent 
mechanism may also serve to augment insulin secretion in the face of increased demands. Rather than the age expected decline in beta cell mass, pancreases from $d b / d b$ mice that had received SRT3025 displayed enlarged insulinimmunopositive islets. To examine possible mechanisms that may account for these salutary findings, we explored changes in the expression of betatrophin, a recently identified hormone that increases beta cell proliferation in response to insulin resistance and hyperglycaemia [22]. Here, we show that hepatic betatrophin expression was increased a further three- to four-fold in SRT3025-treated $d b / d b$ animals. By comparison, SIRT1 activation did not affect betatrophin expression in nondiabetic $d b / m$ mice.

The ability to adapt energy expenditure to nutrient availability is a key survival mechanism. Although there is marked variation among 'warm-blooded' animals, considerable energy is used in the maintenance of body temperature and physical activity [26]. Reduction in both, however, can be achieved by bouts of torpor that may manifest as either hibernation or daily torpor. Hibernation tends to be prolonged, often lasting the entire winter, relying critically on the stored fat that has been accumulated in its preliminary phase. Daily torpor, on the other hand, typically lasts only a few hours, is characterised by lesser reductions in body temperature, relies on energy from ingested food rather than stored fat and can be inhibited by leptin [27]. As expected, $d b /$ $d b$ mice deficient in leptin receptor underwent shallow torpor that extended from the latter part of dark phase to the early light phase, as is typical for nocturnal animals [28]. Consistent with its role in survival during energy deprivation, SIRT1 activation in the present study not only increased the magnitude of temperature reduction but also diminished energy expenditure and activity. While the short duration of these effects is typical of torpor, the fat accumulation that was also noted is more typical of the early phase of hibernation. In $d b / m$ mice, a similar, albeit incomplete response to SIRT1 activation was noted whereby mice that received SRT3025 showed reduced energy expenditure and activity but not body temperature. Although, blood glucose was reduced in diabetic mice, other features that accompanied the administration of SRT3025 may be less desirable in diabetic patients. However, given the importance of genetic background to the development of diabetes in both the animal and human setting, how these findings might translate to human type 2 diabetes, where neither hibernation nor torpor occur, remains to be determined.

Heat production and body temperature are reduced during periods of lower activity and food consumption, ostensibly to conserve energy. In the present study, we observed dramatic changes in the amplitude in the circadian rhythm of body temperature in $d b / d b$ mice treated with SRT3025 when compared with untreated $d b / d b$ mice or $d b / m$ mice that had also received the SIRT1 activator. Together, these findings suggest that while both SIRT1 and leptin are known to modulate circadian rhythms [29-32], when combined their effects may be more than additive.

Though currently viewed as a pathophysiological state of disordered lipid and carbohydrate metabolism with considerable associated morbidity [33], the ability to accumulate fat is a normal physiological process that aids survival and averts starvation in the setting of impending food shortage [34]. Indeed, just prior to hibernation, animals gain substantial body and hepatic fat mass. The accumulation of fat in the livers of SRT3025-treated diabetic mice, as seen in the present study, is consistent with the known ability of SIRT1 to activate Liver X receptor alpha [35], the consequences of which would include increased expression of Srebp 1c [36], Fasn [37] mRNAs and hepatic steatosis [37, 38], as observed in our study. In addition, changes in FOXO1 expression were also noted in the current study with increased Foxol mRNA in $d b / d b$ mice that was reduced with the administration of SRT3025. These alterations would be expected to lead to changes in the liver's major metabolic pathways with a reduction in gluconeogenesis and increased lipogenesis [39], findings consistent with the reduced Pepck and G6pc mRNA, along with the increase in Fasn mRNA and increased RER demonstrated in our study. Importantly, however, while increased lipogenesis and hepatic steatosis were features of $d b / d b$ mice treated with SRT3025, other studies using a similar SIRT1 activating compound, SRT1720, showed reduced fatty liver in diet-induced obese, non-diabetic mice $[40,41]$. These findings highlight the importance of genetic background and metabolic state in modulating the response to pharmacological intervention.

Notably, despite the associated insulin resistance, hibernating animals do not develop hyperglycaemia with its attendant glycosuria and insensible energy loss. Suggestive of transition to this more physiological, torpor-like state, obese insulinresistant $d b / d b$ mice that received the SIRT1 activator SRT3025 in the present study displayed lower plasma glucose concentrations and did not develop the polyuria seen in untreated animals.

Diminished insulin secretion and enhanced insulin resistance together contribute to the pathogenesis of type 2 diabetes in the $d b / d b$ mouse and the vast majority of humans. While SIRT1 activation greatly augmented circulating insulin in the present study, insulin resistance, as indicated by both HOMAIR and IRS-1 phosphorylation, was exacerbated in response to drug administration. These findings are consistent with some, but by no means all, previous studies that have examined the effects of SIRT1 modulation. For instance, Sirt1 overexpression by the systemic administration of adenovirus expressing Sirt1 transcript reduced insulin sensitivity in 6-8-week-old male BALB/c mice [42]. Consistent with this, reducing Sirt1 expression with either small hairpin RNA or antisense 
oligonucleotide improved insulin sensitivity $[42,43]$. By contrast, hepatic Sirt1 deficiency was found to worsen insulin resistance in Sirt ${ }^{\text {flox }}$ albumin-cre mice [44]. Previous studies have also addressed the effects of pharmacological Sirt1 activation in the diabetes setting. For instance, administration of SRT1720 reduced blood glucose in high-fat diet fed, obese $L e p^{o b / o b}$ mice and while they did not gain weight, the mice received the drug for only 1 week [45]. Moreover, in other studies, mice with high-fat feeding-induced metabolic syndrome (but not diabetes), preliminary data suggest that these animals are protected from weight gain and hepatic steatosis with SRT3025 [46, 13].

By responding to metabolic signals such as leptin, insulin and glucose, the orexigenic, neuropeptide Y (NPY)/agoutirelated peptide and thyrotrophin releasing hormone (TRH) neurons of the hypothalamus coordinate changes in food intake and energy expenditure, respectively, that together aim to maintain homeostasis. While reductions in hypothalamic Npy and Trh mRNA and circulating free T3 were seen in $d b / d b$ mice in the present study, levels in these mice were unaffected by Sirt1 activation.

In summary, SIRT1 activation in $d b / d b$ mice led to substantial expansion of beta cell mass, increased circulating insulin and improved glycaemic control. These ostensibly beneficial changes were, in this mouse model of type 2 diabetes, also accompanied by weight gain, reduced physical activity, diminished energy expenditure and hepatic steatosis. While in some ways reminiscent of torpor and hibernation, the summative impact of these changes on the long-term progression of type 2 diabetes remain to be unravelled, particularly in the setting of the complex interaction between Sirt1 modulation and genetic factors, as seen in this study.

Acknowledgements The authors would like to thank J. Switzer, A. Katz and D. Koh (Keenan Research Centre for Biomedical Science and Li Ka Shing Knowledge Institute of St Michael's Hospital, Toronto, ON, Canada) for their technical assistance with animal studies, immunohistochemistry and data management. The authors also thank Sirtris/Glaxo Smith-Kline for their gift of SRT3025.

Funding The studies were supported by a grant from the Canadian Institutes of Health Research (260193). RG is the Canadian Research Chair in Diabetes Complications and this research was supported in part by the Canada Research Chair Program.

Contribution statement RG designed the study, drafted the manuscript and approved its final version. YZ, MW and CLC contributed to the study design, revised the article's intellectual content and approved the final version. KT, SA, DK and SS acquired data, revised the article's intellectual content and approved the final version. RG and $\mathrm{YZ}$ are responsible for the integrity of this work.

Duality of interest RG has received a grant to his institution from Sirtris/GSK. All other authors declare that there is no duality of interest associated with their contribution to this manuscript.

\section{References}

1. Iozzo P, Beck-Nielsen H, Laakso M, Smith U, Yki-Jarvinen H, Ferrannini E (1999) Independent influence of age on basal insulin secretion in nondiabetic humans. European Group for the Study of Insulin Resistance. J Clin Endocrinol Metab 84:863-868

2. Basu R, Breda E, Oberg AL et al (2003) Mechanisms of the ageassociated deterioration in glucose tolerance: contribution of alterations in insulin secretion, action, and clearance. Diabetes 52:17381748

3. Carey HV, Andrews MT, Martin SL (2003) Mammalian hibernation: cellular and molecular responses to depressed metabolism and low temperature. Physiol Rev 83:1153-1181

4. Buck MJ, Squire TL, Andrews MT (2002) Coordinate expression of the PDK4 gene: a means of regulating fuel selection in a hibernating mammal. Physiol Genomics 8:5-13

5. Nelson BT, Ding X, Boney-Montoya J, Gerard RD, Kliewer SA, Andrews MT (2013) Metabolic hormone FGF21 is induced in ground squirrels during hibernation but its overexpression is not sufficient to cause torpor. PLoS One 8:e53574

6. Lin SJ, Defossez PA, Guarente L (2000) Requirement of NAD and SIR2 for life-span extension by calorie restriction in Saccharomyces cerevisiae. Science 289:2126-2128

7. Tissenbaum HA, Guarente L (2001) Increased dosage of a sir-2 gene extends lifespan in Caenorhabditis elegans. Nature 410:227-230

8. Rogina B, Helfand SL (2004) Sir2 mediates longevity in the fly through a pathway related to calorie restriction. Proc Natl Acad Sci U S A 101:15998-16003

9. Yu J, Auwerx J (2009) The role of sirtuins in the control of metabolic homeostasis. Ann N Y Acad Sci 1173(1):E10-E19

10. Lavu S, Boss O, Elliott PJ, Lambert PD (2008) Sirtuins-novel therapeutic targets to treat age-associated diseases. Nat Rev Drug Discov 7:841-853

11. Dai H, Kustigian L, Carney D et al (2010) SIRT1 activation by small molecules: kinetic and biophysical evidence for direct interaction of enzyme and activator. J Biol Chem 285:32695-32703

12. Hubbard BP, Gomes AP, Dai H et al (2013) Evidence for a common mechanism of SIRT1 regulation by allosteric activators. Science 339: 1216-1219

13. Suri V, Davis M, Qi Y, et al (2013) Pharmacological prevention of diet induced metabolic dysfunction by the SIRT1 activator SRT3025. Proceedings of the 73rd Annual Scientific Sessions of the American Diabetes Association, Chicago, June 21-25: 1197-P (Abstract)

14. Cota D, Proulx K, Smith KA et al (2006) Hypothalamic mTOR signaling regulates food intake. Science 312:927-930

15. Nguyen KT, Tajmir P, Lin CH et al (2006) Essential role of Pten in body size determination and pancreatic beta-cell homeostasis in vivo. Mol Cell Biol 26:4511-4518

16. Matthews DR, Hosker JP, Rudenski AS, Naylor BA, Treacher DF, Turner RC (1985) Homeostasis model assessment: insulin resistance and beta-cell function from fasting plasma glucose and insulin concentrations in man. Diabetologia 28:412-419

17. Li L, Hossain MA, Sadat S et al (2011) Lecithin cholesterol acyltransferase null mice are protected from diet-induced obesity and insulin resistance in a gender-specific manner through multiple pathways. J Biol Chem 286:17809-17820

18. Garver J, Bermeo-Blanco OA, Gibson N, Bogie H, Grenwis J, Vela EM (2012) Implantation and monitoring of a novel telemetry unit in the Syrian golden hamster model. J Invest Surg 25:186-196

19. Advani A, Kelly DJ, Advani SL et al (2007) Role of VEGF in maintaining renal structure and function under normotensive and hypertensive conditions. Proc Natl Acad Sci U S A 104:1444814453 
20. Kosanam H, Thai K, Zhang Y et al (2014) Diabetes induces lysine acetylation of intermediary metabolism enzymes in the kidney. Diabetes 63:2432-2439

21. Anstee QM, Goldin RD (2006) Mouse models in non-alcoholic fatty liver disease and steatohepatitis research. Int J Exp Pathol 87:1-16

22. Yi P, Park JS, Melton DA (2013) Betatrophin: a hormone that controls pancreatic beta cell proliferation. Cell 153:747-758

23. Mantzoros CS, Flier JS (2000) Editorial: leptin as a therapeutic agent-trials and tribulations. J Clin Endocrinol Metab 85:4000-4002

24. Moynihan KA, Grimm AA, Plueger MM et al (2005) Increased dosage of mammalian Sir2 in pancreatic beta cells enhances glucose-stimulated insulin secretion in mice. Cell Metab 2:105-117

25. Bordone L, Motta MC, Picard F et al (2006) Sirt1 regulates insulin secretion by repressing UCP2 in pancreatic beta cells. PLoS Biol 4: e31

26. Landsberg L (2012) Core temperature: a forgotten variable in energy expenditure and obesity? Obes Rev 13(Suppl 2):97-104

27. Swoap SJ (2008) The pharmacology and molecular mechanisms underlying temperature regulation and torpor. Biochem Pharmacol $76: 817-824$

28. Geiser F (2004) Metabolic rate and body temperature reduction during hibernation and daily torpor. Annu Rev Physiol 66:239-274

29. Chang HC, Guarente L (2013) SIRT1 mediates central circadian control in the SCN by a mechanism that decays with aging. Cell 153:1448-1460

30. Vinciguerra M, Fulco M, Ladurner A, Sartorelli V, Rosenthal N (2010) SirT1 in muscle physiology and disease: lessons from mouse models. Dis Model Mech 3:298-303

31. Sahar S, Sassone-Corsi P (2013) The epigenetic language of circadian clocks. Handb Exp Pharmacol 217:29-44

32. Wiater MF, Li AJ, Dinh TT, Jansen HT, Ritter S (2013) Leptinsensitive neurons in the arcuate nucleus integrate activity and temperature circadian rhythms and anticipatory responses to food restriction. Am J Physiol Regul Integr Comp Physiol 305:R949-R960

33. Bechmann LP, Hannivoort RA, Gerken G, Hotamisligil GS, Trauner M, Canbay A (2012) The interaction of hepatic lipid and glucose metabolism in liver diseases. J Hepatol 56:952-964

34. Johnson RJ, Stenvinkel P, Martin SL et al (2013) Redefining metabolic syndrome as a fat storage condition based on studies of comparative physiology. Obesity (Silver Spring) 21:659-664
35. Li X, Zhang S, Blander G, Tse JG, Krieger M, Guarente L (2007) SIRT1 deacetylates and positively regulates the nuclear receptor LXR. Mol Cell 28:91-106

36. Repa JJ, Liang G, Ou J et al (2000) Regulation of mouse sterol regulatory element-binding protein-1c gene (SREBP-1c) by oxysterol receptors, LXR $\alpha$ and LXR $\beta$. Genes Dev 14:2819-2830

37. Peet DJ, Turley SD, Ma W et al (1998) Cholesterol and bile acid metabolism are impaired in mice lacking the nuclear oxysterol receptor LXR alpha. Cell 93:693-704

38. Beaven SW, Matveyenko A, Wroblewski K et al (2013) Reciprocal regulation of hepatic and adipose lipogenesis by liver $\mathrm{X}$ receptors in obesity and insulin resistance. Cell Metab 18:106-117

39. Zhang W, Patil S, Chauhan B et al (2006) FoxO1 regulates multiple metabolic pathways in the liver: effects on gluconeogenic, glycolytic, and lipogenic gene expression. J Biol Chem 281:10105-10117

40. Feige JN, Lagouge M, Canto C et al (2008) Specific SIRT1 activation mimics low energy levels and protects against diet-induced metabolic disorders by enhancing fat oxidation. Cell Metab 8:347-358

41. Yamazaki Y, Usui I, Kanatani Y et al (2009) Treatment with SRT1720, a SIRT1 activator, ameliorates fatty liver with reduced expression of lipogenic enzymes in MSG mice. Am J Physiol Endocrinol Metab 297:E1179-E1186

42. Rodgers JT, Puigserver P (2007) Fasting-dependent glucose and lipid metabolic response through hepatic sirtuin 1. Proc Natl Acad Sci U S A 104:12861-12866

43. Erion DM, Yonemitsu S, Nie Y et al (2009) SirT1 knockdown in liver decreases basal hepatic glucose production and increases hepatic insulin responsiveness in diabetic rats. Proc Natl Acad Sci U S A 106:11288-11293

44. Wang RH, Kim HS, Xiao C, Xu X, Gavrilova O, Deng CX (2011) Hepatic Sirt1 deficiency in mice impairs mTorc2/Akt signaling and results in hyperglycemia, oxidative damage, and insulin resistance. J Clin Invest 121:4477-4490

45. Milne JC, Lambert PD, Schenk S et al (2007) Small molecule activators of SIRT1 as therapeutics for the treatment of type 2 diabetes. Nature 450:712-716

46. Nie Q, Qi Y, Davis M et al (2012) SRT3025, a novel small molecule SIRT1 activator, reduces hepatic steatosis in diet induced obese (DIO) mice through suppression of lipogenesis and increased fatty acid oxidation. Diabetes 61(Suppl1):A455 (Abstract) 